\title{
BMJ Open Frequent refresher training on newborn resuscitation and potential impact on perinatal outcome over time in a rural Tanzanian hospital: an observational study
}

\author{
Estomih Mduma, ${ }^{1,2}$ Jan Terje Kvaløy, ${ }^{3,4}$ Eldar Soreide, ${ }^{5,6}$ Erling Svensen, ${ }^{7}$ \\ Paschal Mdoe, ${ }^{1,8}$ Jeffrey Perlman, ${ }^{9}$ Caroline Johnson, ${ }^{10}$ \\ Hussein Lessio Kidanto, ${ }^{11,12}$ Hege Langli Ersdal ${ }^{13,14}$
}

To cite: Mduma E, Kvaløy JT, Soreide $\mathrm{E}$, et al. Frequent refresher training on newborn resuscitation and potential impact on perinatal outcome over time in a rural Tanzanian hospital: an observational study. BMJ Open 2019;9:e030572. doi:10.1136/ bmjopen-2019-030572

- Prepublication history for this paper is available online. To view these files, please visit the journal online (http://dx.doi. org/10.1136/bmjopen-2019030572).

Received 23 April 2019

Revised 04 September 2019

Accepted 05 September 2019

Check for updates

(C) Author(s) (or their employer(s)) 2019. Re-use permitted under CC BY-NC. No commercial re-use. See rights and permissions. Published by BMJ.

For numbered affiliations see end of article.

Correspondence to

Dr Estomih Mduma; estomduma@gmail.com

\section{ABSTRACT}

Objectives Globally, perinatal mortality remains high, especially in sub-Saharan countries, mainly because of inadequate obstetric and newborn care. Helping Babies Breathe (HBB) resuscitation training as part of a continuous quality improvement (CQl) programme may improve outcomes. The aim of this study was to describe observed changes in perinatal survival during a 6-year period, while adjusting for relevant perinatal risk factors. Setting Delivery rooms and operating theatre in a rural referral hospital in northern-central Tanzania providing comprehensive obstetric and basic newborn care 24 hours a day. The hospital serves approximately 2 million people comprising low social-economic status.

Participants All newborns ( $n=31122)$ born in the hospital from February 2010 through January 2017; 4893 were born in the 1-year baseline period (February 2010 through January 2011), 26229 in the following CQI period. Interventions The HBB CQI project, including frequent HBB training, was implemented from February 2011. This is a quality assessment analysis of prospectively collected observational data including patient, process and outcome measures of every delivery. Logistic regression modelling was used to construct risk-adjusted variable life adjusted display (VLAD) and cumulative sum (CUSUM) plots to monitor changes in perinatal survival (primary outcome). Results During the 6-year CQI period, the unadjusted number of extra lives saved according to the VLAD plot was 150 despite more women admitted with pregnancy and labour complications and more caesarean deliveries. After adjusting for these risk factors, the risk-adjusted VLAD plot indicated that an estimated 250 extra lives were saved. The risk-adjusted CUSUM plot confirmed a persistent and steady increase in perinatal survival. Conclusions The risk-adjusted statistical process control methods indicate significant improvement in perinatal survival after initiation of the HBB CQI project with continuous focus on newborn resuscitation training during the period, despite a concomitant increase in high-risk deliveries. Risk-adjusted VLAD and CUSUM are useful methods to quantify, illustrate and demonstrate persistent changes in outcome over time.
Strengths and limitations of this study

- The prospective and detailed data collection by observers, not taking part in the care of mothers and babies, is a major strength of this study. Previous studies have shown that quality improvement studies based on self-reporting by involved staff may be flawed.

The long duration of the study, 7 years, made it possible to follow several annual seasonal changes, adding strength to the study.

- The high number of newborns observed in the cohort with complete data set makes the findings from this study more convincing.

- The study had an observational design and was conducted in one setting, both limiting generalisation of the findings.

- There were several randomised controlled studies conducted during the study period, evaluating medical devices for fetal heart rate monitoring and newborn ventilation. These studies could have influenced our findings, however, none of them showed any significant impact on perinatal survival.

\section{INTRODUCTION}

Globally, there have been considerable efforts to reduce the under- 5 years child mortality, mostly occurring in low-resource countries. These joint efforts have resulted in an approximately $56 \%$ reduction in the under-5 years mortality between 1990 and 2016 , from 12.7 million to 5.6 million deaths. ${ }^{1-4}$ However, despite numerous efforts, newborn mortality has not decreased in a similar way, and currently contributes to approximately $46 \%$ of the under-5 years mortality, which reflects an increase of about $41 \%$ from $2000 .^{5}$ This translates into approximately 7000 newborns dying every day, with the highest burden in sub-Saharan Africa. ${ }^{56}$ Additionally, 
an estimated 2.6 million fresh stillbirths, that is, intrapartum-related deaths, were reported in $2016^{78}$ making the burden of early perinatal mortality (ePMR), that is, fresh stillbirths and 24hours' newborn deaths, a huge challenge. Importantly, most of these deaths are secondary to potentially preventable causes of birth asphyxia, with disruption of placental blood flow, as the most prominent contributing factor. ${ }^{6-10}$

In 2009, Tanzania was selected to pilot test a new simulation-based training curriculum for newborn resuscitation called Helping Babies Breathe (HBB), ${ }^{11}$ to reduce ePMR. Haydom Lutheran Hospital (HLH), a rural referral hospital in northern-central Tanzania, was selected as one of eight study sites, with data collection starting in February 2010. ${ }^{11}$ Over the subsequent years, efforts to identify factors contributing to perinatal mortality and strategies to reduce perinatal deaths, especially from birth asphyxia, have been implemented at $\mathrm{HLH}^{10-15}$ and in similar low-resource settings. ${ }^{16}{ }^{17}$ In February 2011, a continuous quality improvement (CQI) programme was initiated with enforced focus on HBB newborn resuscitation refresher training. ${ }^{12}$ Building on the CQI programme focusing on frequent HBB training, the 'Safer Births' project was initiated in 2013, including development and testing of new training and treatment equipment for fetal heart rate monitoring during labour and newborn resuscitation. ${ }^{15}$ In 2016, a newborn ventilation trainer system (Laerdal Global Health) for onsite refresher training was introduced. This system was designed to be easily incorporated with the HBB training, comprising automatic training feedback, with the goal to further stimulate frequent $\mathrm{HBB}$ scenario training involving all relevant staff.

Using statistical process control (SPC) methods, we recently reported an improved perinatal survival rate from 2011 to 2016 with an estimated 120 extra lives saved in this period at HLH. ${ }^{15}$ We also described the different exposures and/or interventions occurring over the time period, which could have had an impact on perinatal outcome. Most trends of improved survival in the applied cumulative sum (CUSUM) plot matched with enforced focus on newborn resuscitation training. ${ }^{15} \mathrm{SPC}$ methods like CUSUM and variable life adjusted display (VLAD) are well suited for detecting and quantifying small persistent changes in perinatal outcome over time. ${ }^{1819}$

The aim of this study was to assess whether a persistent increase in perinatal survival over 6 years following introduction of an HBB CQI programme could be detected when adjusting for changes in perinatal risk factors. A limitation with the previous work was that possible changes in patient risk factors for ePMR within the cohort over time were not adjusted for.

\section{METHODS \\ Setting}

This is a retrospective analysis of data from a prospective observational study conducted at HLH, a rural referral hospital located in northern-central Tanzania from February 2010 through January 2017. The baseline ePMR was $2.7 \%$ ( $\mathrm{n}=133$ deaths). The catchment area for HLH is approximately 2 million people comprising predominantly low social economic status. ${ }^{20}$ HLH provides comprehensive obstetric and basic newborn care 24 hours a day, 7 days a week. The labour ward has six delivery rooms with one delivery bed each, and one operating theatre where caesarean sections (CS) take place. Data were collected from all delivery rooms and the operating theatre.

\section{Newborn resuscitation training interventions during the study period}

Figure 1 presents different interventions and events during the study period.

\section{One-year baseline period: February 2010 through January 2011}

Data collection for the National HBB study started in February 2010. HBB consisted of practical training on basic newborn care and resuscitation. ${ }^{10-12} 15$ One full-day HBB training was conducted in April 2010, facilitated by master trainers from the Tanzanian Ministry of Health. However, not all relevant staff were trained and no CQI efforts were introduced after the training. Evaluation 7 months after this 1 -day HBB training revealed no

\begin{tabular}{|c|c|c|c|c|c|c|c|c|c|c|c|c|c|c|c|c|c|c|c|c|c|c|c|c|c|c|c|c|c|c|c|}
\hline \multirow[b]{2}{*}{ Interventions and events } & \multicolumn{4}{|c|}{2009} & \multicolumn{4}{|c|}{2010} & \multicolumn{4}{|c|}{2011} & \multicolumn{4}{|c|}{2012} & \multicolumn{4}{|c|}{2013} & \multicolumn{4}{|c|}{2014} & \multicolumn{3}{|c|}{2015} & \multicolumn{4}{|c|}{2016} \\
\hline & q1 & $\mathrm{q} 2$ & q3 & q4 & q1 & q2 $\mathrm{c}$ & q3 & $\mathrm{q} 4$ & q1 & $\mathrm{q} 2$ & $\mathrm{q3}$ & q4 & q1 & $\mathrm{q} 2$ & q3 & $q 4$ & $\mathrm{q} 1$ & $\mathrm{q} 2$ & \begin{tabular}{l|l}
3 & $q$ \\
$q$
\end{tabular} & 94 & \begin{tabular}{l|l}
$\mathrm{q} 1 \mathrm{q}$ \\
$\mathrm{a}$
\end{tabular} & $\mathrm{q} 2^{2} \mathrm{q3}$ & \begin{tabular}{l|l}
3 & $q^{4}$ \\
\end{tabular} & $\begin{array}{l}4 \mathrm{q} \\
\mathrm{a}\end{array}$ & $\begin{array}{ll}\mathrm{q} & \mathrm{q} \\
\mathrm{a}\end{array}$ & \begin{tabular}{|l|l}
2 & $q^{3}$ \\
\end{tabular} & \begin{tabular}{|l|l}
4 \\
\end{tabular} & $\mathrm{q} 1$ & \begin{tabular}{|l|}
$\mathrm{q} 2$ \\
\end{tabular} & $\mathrm{q} 3$ & $\mathrm{q} 4$ \\
\hline Observation in the delivery room & & & & & & & & & & & & & & & & & & & & & & & & & & & & & & & \\
\hline Baseline HBB CQI (Feb 10-Feb 11) & & & & & & & & & & & & & & & & & & & & & & & & & & & & & & & \\
\hline One-day HBB training & & & & & & & & & & & & & & & & & & & & & & & & & & & & & & & \\
\hline HBB CQI with frequent training & & & & & & & & & & & & & & & & & & & & & & & & & & & & & & & \\
\hline Training local Master Trainers & & & & & & & & & & & & & & & & & & & & & & & & & & & & & & & \\
\hline Newborn Ventilation Trainer & & & & & & & & & & & & & & & & & & & & & & & & & & & & & & & \\
\hline & & & & & & & & & & & & & & & & & & & & & & & & & & & & & & & \\
\hline Safer Births activities & & & & & & & & & & & & & & & & & & & & & & & & & & & & & & & \\
\hline Instalment of Monitors & & & & & & & & & & & & & & & & & & & & & & & & & & & & & & & \\
\hline Fetal heart rate RCT 1 & & & & & & & & & & & & & & & & & & & & & & & & & & & & & & & \\
\hline Fetal heart rate RCT 2 & & & & & & & & & & & & & & & & & & & & & & & & & & & & & & & \\
\hline Newborn ventilation $R C T$ & & & & & & & & & & & & & & & & & & & & & & & & & & & & & & & \\
\hline Premature Bundle Study & & & & & & & & & & & & & & & & & & & & & & & & & & & & & & & \\
\hline & & & & & & & & & & & & & & & & & & & & & & & & & & & & & & & \\
\hline Administrative events & & & & & & & & & & & & & & & & & & & & & & & & & & & & & & & \\
\hline Experienced staff leaving & & & & & & & & & & & & & & & & & & & & & & & & & & & & & & & \\
\hline New staff recruited & & & & & & & & & & & & & & & & & & & & & & & & & & & & & & & \\
\hline Ambulance fee & & & & & & & & & & & & & & & & & & & & & & & & & & & & & & & \\
\hline Delivery fee & & & & & & & & & & & & & & & & & & & & & & & & & & & & & & & \\
\hline
\end{tabular}

Figure 1 Overview of different interventions and events during the study period. CQI, continuous quality improvement; HBB, Helping Babies Breathe; RCT, randomised controlled trial. 
changes in clinical management, ${ }^{21}$ leading to initiation of the HBB CQI programme in 2011.

\section{Introduction of the HBB CQI programme in 2011 and Safer Births} in 2013

Due to lack of improvement in clinical management in the delivery room, ${ }^{21}$ a programme encouraging frequent brief onsite simulation HBB trainings among the midwives was implemented in February 2011 (HBB CQI). Five local midwives were trained to become HBB trainers, with the responsibility to facilitate ongoing frequent HBB trainings in the labour ward. ${ }^{12}$ Short $(5-10 \mathrm{~min})$ mandatory HBB simulation-based training sessions were conducted on a weekly basis over the following 6 years.

In early 2013, the Safer Births project was initiated, which included instalment of newborn resuscitation monitors (Laerdal Global Health, Stavanger, Norway). The newborn heart rate was displayed on the monitor as a continuous feedback to the provider. In 2016, a newborn ventilation trainer system (Laerdal Global Health) for low-dose high-frequency onsite practice was introduced. The training involved the use of a novel newborn manikin (NeoNatalie Advanced Prototype, Laerdal Global Health) which could be adjusted to simulate four different common resuscitation scenarios. These were based on real data from more than 1000 live resuscitations observed and recorded by the newborn resuscitation monitor at HLH. The training system was easily operated using a tablet providing an immediate feedback to the provider after a training session, with specific tips to improve. The training system facilitated both individual skills and scenario team training.

\section{Other research and administrative exposures during the study period}

Research on fetal heart rate monitoring during labour

As part of Safer Births, two randomised controlled studies comparing different devices for fetal heart rate monitoring, involving low-risk deliveries, were conducted at HLH. $^{22}{ }^{23}$ The first study, from March 2013 to August 2015, compared a wind-up handheld Doppler (FreePlay) and the Pinard fetoscope (commonly used in this setting). ${ }^{22}$ The second study, from February 2016 to January 2017, compared a new strap-on continuous fetal heart rate monitor named Moyo (Laerdal Global Health) and the Pinard fetoscope for intermittent monitoring. ${ }^{23}$ Moyo is a robust low-cost device developed for low-resource settings, reported to improve midwifery care. ${ }^{24}$ No significant changes in ePMR outcomes were reported in the two randomised controlled studies at HLH. ${ }^{22} 23$

\section{Research on newborn care and resuscitation}

Between October 2014 and June 2016, a randomised controlled study comparing the standard newborn resuscitator (Laerdal Medical) with a new upright resuscitator (Laerdal Global Health) for ventilation of non-breathing newborns was conducted at HLH. ${ }^{25}$ This study included additional training on bag mask ventilation skills. No significant changes in ePMR were reported during the study period. ${ }^{25}$

In 2014-2017, HLH took part in a premature multicentre study led by the Ministry of Health, including premature newborns less than 34 weeks' gestation. ${ }^{26} \mathrm{~A}$ bundle-of-care approach (ie, antenatal corticosteroids, maternal and newborn antibiotics, immediate HBB intervention and avoidance of hypothermia) was introduced, but no significant change in newborn mortality was reported at HLH. ${ }^{26}$

\section{Administrative exposures}

During the reference study period (2011-2017), a high turnover of midwives was noted, particularly in relation to new government employment opportunities every midyear (experienced HLH staff leaving to be employed in other government-owned health facilities) and towards the end of each year (HLH recruited new midwives who had completed midwifery training at Haydom School of Nursing to fill the gaps).

An ambulance fee was introduced in July 2013 and a delivery fee in January 2014.

\section{Data collection and management}

Trained research assistants have observed every delivery in the labour ward, working 2-3 in each shift covering 24 hours a day, 7 days a week, using a structured data collection form. The observations started in July 2009, 6 months before the National HBB study. ${ }^{11}$ In this period the staff became familiar to the observers (minimising the Hawthorne effect) and the research assistants were intensively trained in live observations and accurate data collection and reporting. Data collection for this study took place from February 2010 through January 2017. Information collected included pregnancy complication, labour process and outcome, newborn information and birth attendant information. Additionally, to facilitate electronic physiological data collection, newborn resuscitation monitors (Laerdal Global Health), connected to a dry electrode ECG sensor for rapid heart rate detection and a self-inflating bag mask for newborn ventilation, were installed in every delivery room, including the operating theatre where CS took place from March 2013. Data were collected prospectively during this study period, and there was a data quality control system to ensure the validity.

\section{Patient and public involvement}

This study was undertaken in a rural setting, comprising a poor population with a high illiteracy rate and little infrastructure. In such settings, involvement of patients and public is particularly difficult and demanding. However, several individual projects during the study period, like the randomised studies, actively involved the patients. Furthermore, our results are continuously shared through community meetings and community leaders' meetings. The published paper will be located in the hospital library where the community has access. 


\section{Ethical consideration}

This study was approved by the National Institute for Medical Research (NIMR) and the Ministry of Health in Tanzania (the HBB CQI programme Ref NIMR/ HQ/R.8a/Vol IX/1247 and the Safer Births project Ref NIMR/HQ/R8a/Vol IX/1434), and by the Regional Committee for Medical and Health Research Ethics, Western Norway (Ref 2009/302 and Ref number 2013/110/REK). All relevant healthcare providers were informed about the different HBB CQI and Safer Births quality assessment studies and gave oral consent. Patients were also informed about ongoing studies. Oral consents were obtained for participation in the randomised controlled studies. For the quality assessment studies, patient consents were not obtained as approved by the ethical committees.

\section{Data analysis}

Basic count data are presented as numbers and percentages and continuous data as means and SDs. The aim of this study was to monitor and document changes in perinatal survival over time while adjusting for relevant risk factors within the cohort. Therefore, perinatal characteristics and risk factors not related to clinical management were included as explanatory variables in a logistic regression modelling. These risk factors were: birth weight, gestational age, fetal heart rate status, pregnancy complication, fetal presentation of the newborn, multiple birth, source of admission, maternal infection, delivery mode, pre-eclampsia, uterine rupture, cord prolapse and bleeding before labour. First, univariable logistic regression models with each of the listed potential risk factors for perinatal mortality as explanatory variable and perinatal survival as response were fitted. Those risk factors with a $\mathrm{p}$ value $<0.2$ in the univariable model were included in the multivariable modelling. Then a stepwise model selection procedure was run, and finally, removed variables were reintroduced one by one and kept if found significant. Goodness of fit was verified by the Hosmer-Lemeshow test. The regression model was fitted based on the data in the baseline period.

For the data after the baseline period, we constructed a risk-adjusted VLAD plot, ${ }^{27}$ presenting the CUSUM of expected outcome for each newborn if the baseline situation had persisted, minus the observed outcome. The expected outcome is the probability of death according to the logistic regression model. The observed outcome is numbered 0 for survival and 1 for death. The VLAD plot can then be interpreted as the cumulative excess number of survivors over time, compared with the baseline rate taking into account risk factors. For comparison we also made a VLAD plot without the risk adjustment.

Moreover, as a formal statistical monitoring procedure with a signal limit to detect persistent changes, a risk-adjusted CUSUM based on the same logistic regression model as the VLAD plot was constructed. ${ }^{28-30}$ The CUSUM was constructed to quickly detect an improvement of 0.5 percentage points in the ePMR from the baseline level.
The signal limit of this CUSUM was calculated such that with no change in the true survival probability there would on average be one false alarm every 100 months (ie, if there is no change in the true survival probability the CUSUM would remain close to zero and only go above the signal limit on average once per 100 months). The calculations of the CUSUM were done using methods implemented in the R package spcadjust. ${ }^{31}$ For comparison we also made a CUSUM plot without the risk adjustment. Since the aim of this study was to document the impact of improved management on early perinatal (ie, fresh stillbirths and 24 hours' newborn deaths) survival, macerated stillbirths were not included in the regression model and the SPC analyses.

\section{RESULTS}

A total number of 31122 newborns were observed during the time period of February 2010 through January 2017. The number of newborns included in the VLAD and CUSUM analyses (excluding macerated stillbirths and one case with missing outcome data) was 30718 . Of these, 4844 newborns were from the 1-year baseline period (February 2010 through January 2011). Table 1 presents the number of newborns, as well as labour and newborn characteristic distributions across the 7 years' period. The yearly numbers of delivered newborns from 2010 through 2013 were higher (range 4787-4893) compared with the later period from 2014 through 2016 (range 3731-4296). The percentage of babies with abnormal fetal heart rate measurements averaged 2.8\% from 2010 to 2013, then increased to an average of $4.8 \%$ in the following last 3 years (2014-2016). The percentage of fetal heart rate cases that were not measured was also substantially higher in the last 3 years (2014-2016) (average 12.1\%) from an average of $3.5 \%$ for years 2010-2013 (table 1). The proportion of babies delivered vaginally was higher in the first 3 years, and the proportion of cases with labour complications was higher in the later period resulting in the proportion of CS being higher in the last 4 years $(21 \%-23 \%)$ compared with before, that is, 2010-2012 (11\%-15\%). There was an increase in newborns being stimulated after birth in 2015 and $2016(>28 \%)$ compared with before, that is, 2010$2014(<16.5 \%)$, but the number receiving bag mask ventilation was relatively constant over the years. The mean birth weights over the last 3 years were higher (average $3282 \mathrm{~g}$ ) compared with the previous 4years (average 3113 g).

The VLAD plot without risk adjustment shown in figure $2 \mathrm{~A}$ indicates that the number of excess survivors was about 150 in the 6 -year period following implementation of the HBB CQI training programme (2011-2017) as compared with the baseline ePMR (2010-2011). This amounts to a reduction in the ePMR from $2.7 \%$ in the baseline period to $2.2 \%$ in the following 6-year period. There are fluctuations in the curve indicating reduced survival during some of the periods, for example, in August 2011, August 2012, between November 2012 and 


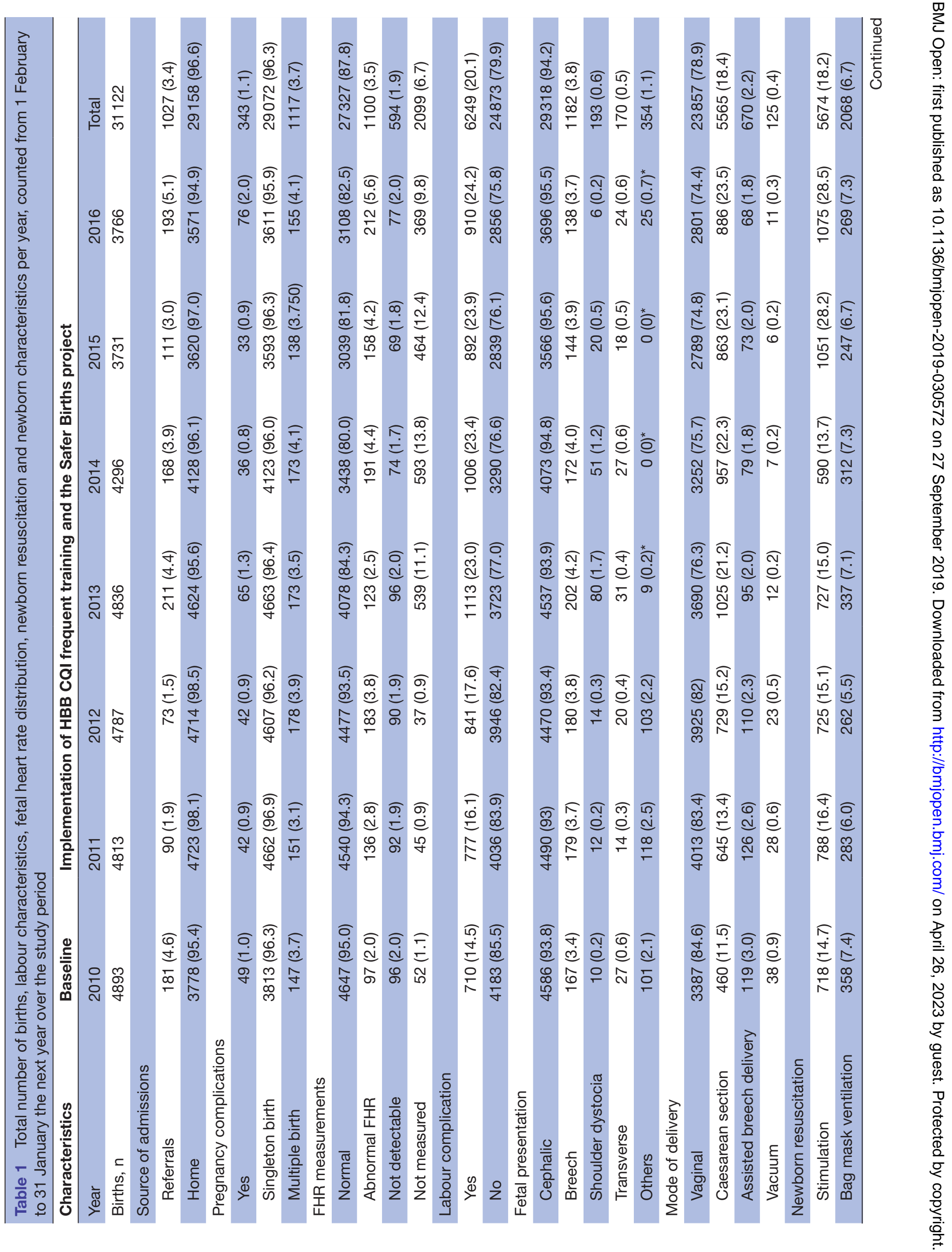


January 2013, in December 2014, and between September and December 2016 (figure 2A).

The significant risk factors included in the logistic regression model for risk adjustment were birth weight $(\mathrm{kg})$, pregnancy complication (yes or no), fetal heart rate (categorised as normal (ie, 120-160 beats per minute), abnormal $(<120$ or $>160$ beats per minute), not detectable, or not measured) and fetal presentation during birth (categorised as cephalic, breech, shoulder dystocia, transverse, or others) (table 2). The risk-adjusted VLAD plot, that is, the calculated number of extra lives saved after adjustment for risk factors, shows a smoother curve, progressively rising, indicating an estimated 250 extra lives saved after risk adjustment (figure 2B). This means that with adjustments for increasing risk factors in the cohort, a calculated 250 extra lives have been saved during the 6-year period, as compared with the baseline period, likely because of improved newborn resuscitation practice.

The unadjusted CUSUM plot signals a persistent improvement after a few months (figure 3A), which confirms that the improvement seen in the VLAD plot is not due to chance. The risk-adjusted CUSUM plot for increased survival crosses the signal limit line earlier and raises more steeply compared with the unadjusted plot (figure 3B).

The risk adjustment had a substantial impact on both the VLAD and the CUSUM plots (figures 2B and 3B), compared with the unadjusted plots (figures $2 \mathrm{~A}$ and 3A). When adjusting for perinatal risk factors, both plots presented a smoother continuous upward curve with very minimal fluctuation. By excluding one variable at a time from the risk-adjusted model, adjustment for abnormal fetal heart rate was the most important factor for the differences between the adjusted and the unadjusted plots (table 2).

\section{DISCUSSION}

The major finding in this study is that the observed increased perinatal survival, following introduction of the CQI programme including frequent HBB simulation-based resuscitation training, was even higher when adjusting for changes in perinatal risk factors over time than what we recently published with no risk adjustments. ${ }^{15}$ With risk adjustments, a calculated 250 extra lives have been saved during the 6-year period as compared with the baseline period. We found that the most influential risk factor for mortality was the increase in newborns with abnormal or not measured fetal heart rate, an observation in concordance to that reported by Langli Ersdal et al. $^{13}$

During the period 2013-2016, the number of women giving birth at HLH progressively decreased from around 4800 annually (during the years 2010-2013) to less than 4300 (in 2014) and to around 3750 (in 2015 and 2016). This progressive reduction appears to be related to the introduction of ambulance (2013) and delivery fees 
A

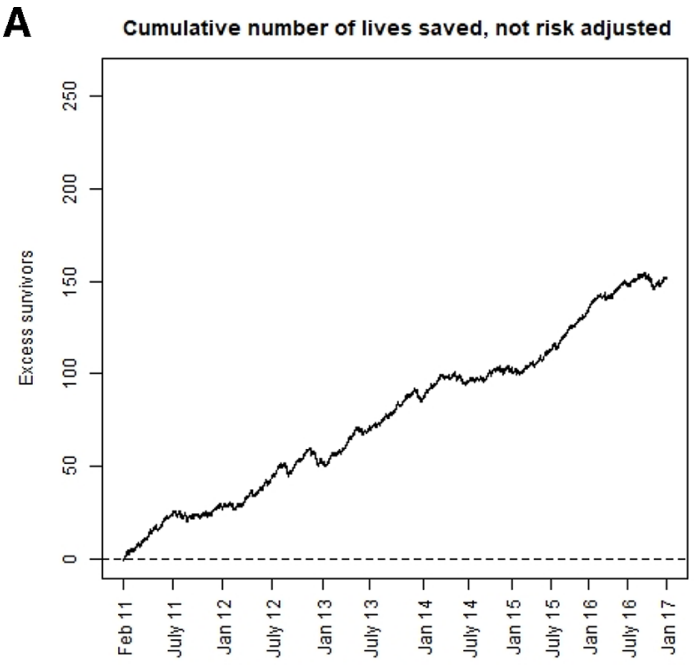

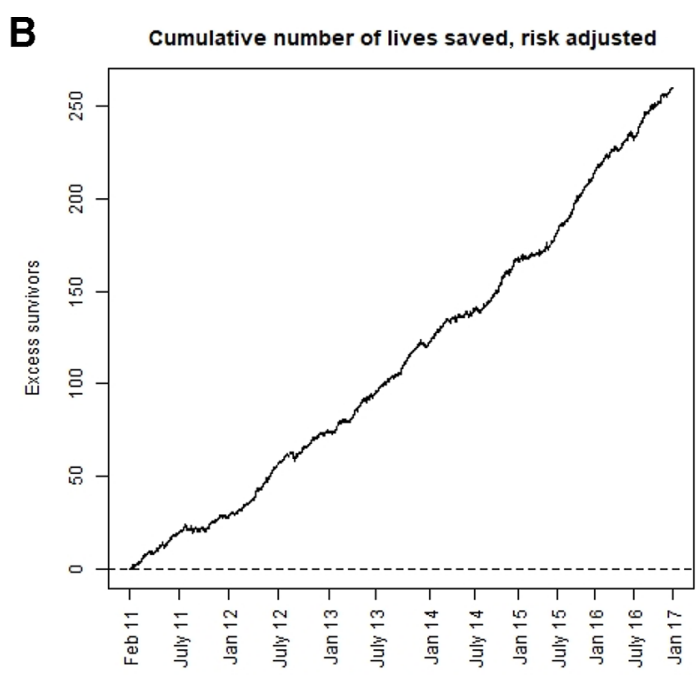

Figure 2 Variable life adjusted display (VLAD) plots displaying the cumulative number of lives saved. (A) Without risk adjustment. (B) With risk adjustment (for birth weight, pregnancy complication, fetal heart rate and fetal presentation).

(2014). Both the ambulance and delivery fees were added to the costs for hospitalisation and hospital management. During the study period before 2013, both ambulance and all delivery services were provided at no cost. The population in this catchment area is scattered with long distances to HLH and with limited means of transport, necessitating the need for ambulance transport of women in labour to HLH. Since the community mostly consists of low social economic status, the reduction in number of births is likely associated with the added economical burden related to the fees, which concur with a qualitative study done by Cephas Sialubanje et al in Zambia. This study found that one important reason for home delivery and use of traditional birth attendants was lack of money. ${ }^{32}$ We speculate that more women were forced

Table 2 Logistic regression model for the risk-adjusted VLAD and CUSUM

\begin{tabular}{llll}
\hline Variable & OR & 95\% Cl & P value \\
\hline Birth weight $(\mathrm{Kg})$ & 0.34 & $(0.23$ to 0.51$)$ & $<0.001$ \\
\hline $\begin{array}{l}\text { Pregnancy } \\
\text { complications }\end{array}$ & 3.8 & $(1.1$ to 12.9$)$ & 0.003 \\
$\begin{array}{l}\text { Fetal heart rate } \\
\quad \text { Normal }\end{array}$ & 1 & & $<0.001$ \\
\hline Abnormal & 34.6 & $(19.8$ to 60.3$)$ & $<0.001$ \\
\hline Not detectable & 1289 & $(419$ to 3966$)$ & $<0.001$ \\
\hline Not measured & 5.6 & $(1.6$ to 19.6$)$ & 0.007 \\
\hline Presentation & & & $<0.001$ \\
\hline Cephalic & 1 & & Reference \\
\hline Breech & 2.4 & $(1.02$ to 5.57$)$ & 0.045 \\
\hline Shoulder dystocia & 0.33 & $(0.002$ to 45$)$ & 0.66 \\
\hline Transverse & 8.0 & $(2.4$ to 27$)$ & $<0.001$ \\
\hline Others & 1.5 & $(0.46$ to 4.9$)$ & 0.50 \\
\hline
\end{tabular}

CUSUM, cumulative sum; VLAD, variable life adjusted display. to deliver at home, only proceeding to the hospital if the labour turned out to be complicated. This indicates a more vulnerable cohort from 2013 onwards.

Over the study period, several changes were observed in potential perinatal risk factors for mortality during labour. First, there was a significant increase in the number of cases where the fetal heart rate was not measured, that is, from about $1 \%$ between 2010 and 2012, to above 9\% in 2013-2016, likely indicating that more women were admitted very late in labour with limited time for fetal heart rate assessment. This may be a consequence of more referrals, often admitted in late labour and/or with severe complications. We also speculate that this is as a result of the above-mentioned fees, making women hesitate to call for an ambulance, and thereby arriving late with complications. Second, the number of CS increased from around $15 \%$ in $2010-2012$ to above $21 \%$ in $2013-$ 2016 , likely as a result of increased cases with an abnormal fetal heart rate and/or labour complications. In addition, there was an increase in referred cases from other health facilities during the same period. The increase in CS can also be seen as one of the changes in management, which potentially had a positive impact on ePMR.

The risk-adjusted modelling quantified the level of risk related to each risk factor included in the final model (table 2). The most important risk factor for ePMR was associated with the fetal heart rate, either not detected, not measured or abnormal. We have recently reported that an abnormal fetal heart rate is strongly associated with fresh stillbirths and severely asphyxiated infants. ${ }^{33}$ Fetal heart rate abnormalities often indicate fetal hypoxia, raising the possibility that a fetus may require urgent interventions such as intrauterine resuscitation or an expedited delivery. This may translate into the requirement for immediate delivery room resuscitation if the newborn presents without respirations. The number of newborns who received bag mask ventilation remained constant throughout the study period, however newborns 
A

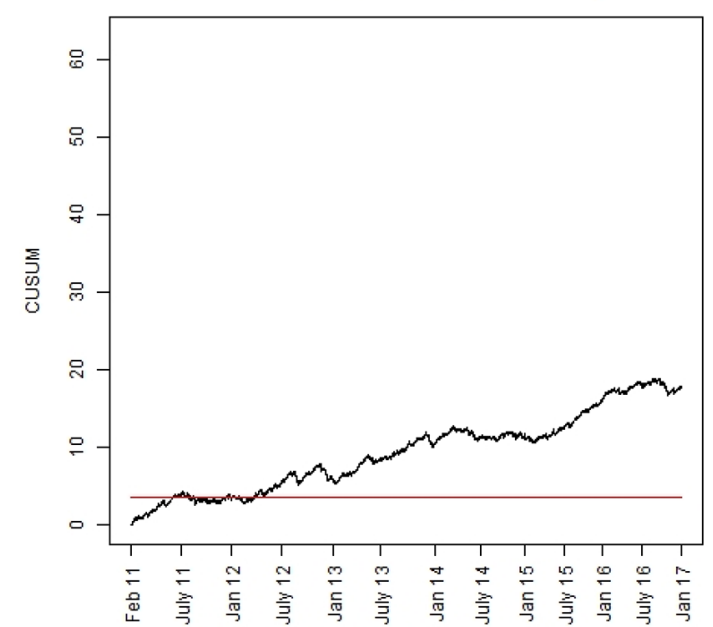

B

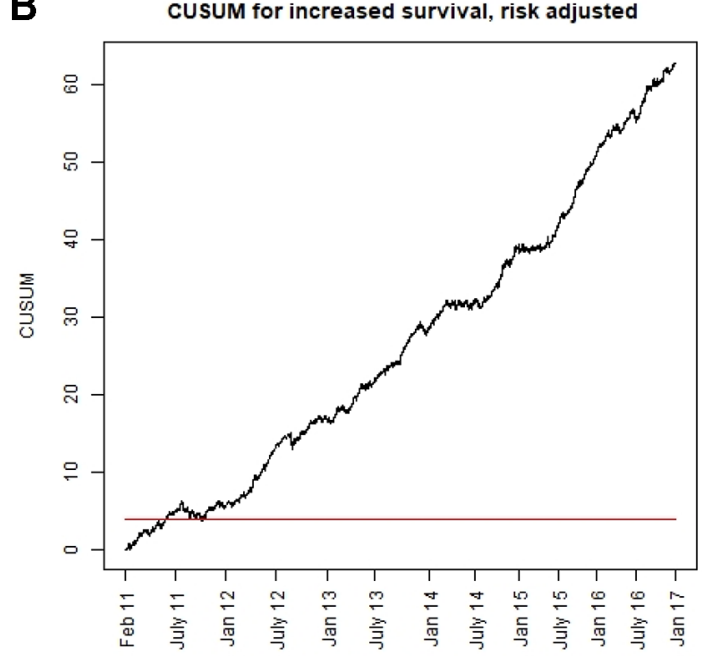

Figure 3 Cumulative sum (CUSUM) for increased perinatal survival. (A) Without risk adjustment. (B) With risk adjustment (for birth weight, pregnancy complication, fetal heart rate and fetal presentation).

being stimulated increased substantially from around $16 \%$ in 2010-2014 to above $28 \%$ in 2015-2016. This likely is a consequence of intensified resuscitation trainings in 2015 and 2016. Thus, the training focused on immediate stimulation prior to initiating bag mask resuscitation. This simple manoeuvre invariably results in the onset of spontaneous breathing, and thus a relatively lesser need for bag mask ventilation, a finding consistent with that of Msemo et al. ${ }^{11}$ The HBB refresher training involved all the staff responsible for attending births/resuscitations, reinforcing the existing frequent brief training programme, which we have shown to be associated with a reduction in mortality. ${ }^{12}$ This addresses the concern raised by Makene et $a l$ and Eblovi et al on the necessity for on-job practice and strengthening of supportive supervision to ensure quality in resuscitation. ${ }^{345}$

Other influences during the study period could also potentially explain the observed changes in perinatal risk factors. The recorded increase in abnormal fetal heart rate could be related to the randomised controlled studies aiming at early detection of abnormal fetal heart rate, ${ }^{22} 23$ however, we consider this influence to be minimal since the studies only involved a subset of the cohort and mostly low-risk cases arriving in early labour.

This study indicates a persistent reduction in ePMR after introducing an HBB CQI programme, in spite of an observed increase in high-risk perinatal cases, especially in the last 3years (2014-2016) of the project. This finding could imply that the newborn management was even better in the 3 last years and indicates the importance of using risk-adjusted models for more reliable estimates.

\section{Strengths and limitations}

A major strength of this study is the prospective and detailed collection of data by observers not taking part in the care of the mothers and babies. Earlier studies have shown that CQI based on self-reporting by involved staff may be flawed. ${ }^{36}$ The study was done in one setting, which is a limitation for generalising the findings. Additional limitations include some changes in practice not directly related to the HBB CQI, such as increased CS over time that may also have influenced perinatal outcome. However, the duration of the study, which was approximately 7 years, and the high number of newborns observed in the cohort with complete data set, and the continuity of the HBB CQI during the entire period make the findings from this study more convincing. Finally, during the last part of the study period several randomised controlled trials were conducted testing different equipment for fetal heart rate monitoring and newborn ventilation. These studies may have influenced the findings, however, none of them showed any significant individual impact on perinatal survival.

\section{CONCLUSION}

To the best of our knowledge, this is the first time risk-adjusted SPC models have been used to estimate effects on ePMR over several years after implementation of an HBB CQI project in a rural sub-Saharan African hospital. The findings indicate a significant improvement in perinatal survival over time, despite a concomitant increase in highrisk deliveries. The estimated number of newborns saved is approximately $40 \%$ higher when adjusting for changes in perinatal risk factors than with no risk adjustment. The HBB CQI programme focusing on short refresher HBB training was the only persisting effort throughout the whole period and seems to be the most important factor for improved perinatal survival, despite an increase in high-risk deliveries.

Risk-adjusted VLAD and CUSUM are useful methods to quantify, illustrate and document reliable persistent changes in outcome over time.

Author affiliations

${ }^{1}$ Haydom Lutheran Hospital, Haydom, Tanzania 
${ }^{2}$ Department of Health, Faculty of Health Sciences, University of Stavanger, Stavanger, Norway

${ }^{3}$ Research, Stavanger University Hospital, Stavanger, Norway

${ }^{4}$ Department of Mathematics and Physics, University of Stavanger Department of Mathematics and Natural Science, Stavanger, Norway

${ }^{5}$ Critical Care and Anaesthesiology Research Group, Department of Clinical Medicine, Stavanger University Hospital, Stavanger, Norway

${ }^{6}$ Faculty of Medicine, University of Bergen, Bergen, Norway

${ }^{7}$ Haukeland Universitetssjukehus, Bergen, Norway

${ }^{8}$ Faculty of Social Science, University of Stavanger, Stavanger, Norway

${ }^{9}$ Pediatrics, Weill Cornell Medical College, New York Presbyterian Hospital, New York City, New York, USA

${ }^{10}$ Department of Mathematics and Natural Science, University of Stavanger,

Stavanger, Norway

${ }^{11}$ School of Medicine, Aga Khan University, Dar es Salaam, Tanzania

${ }^{12}$ Helse Stavanger HF, Stavanger, Norway

${ }^{13}$ Department of Anaesthesiology and Intensive Care, Stavanger University Hospital, Stavanger, Norway

${ }^{14}$ Faculty of Health Sciences, University of Stavanger, Stavanger, Norway

Acknowledgements The authors acknowledge the women who gave birth at Haydom Hospital during the study period, the maternity staff including the supervisors Sara Lyanga and Fortunata Francis, the research staff including Anita Yeconia who supervised the data collection, and the hospital management for allowing this study.

Contributors EM: concept development, study design, data analysis, interpretation of data for the work and manuscript writing. JTK: study design, concept development, data analysis, interpretation of data for the work and critical revision for important intellectual content. ElS: study design, concept development, collection of data, analysis and critical revision for important intellectual content. ErS: study design, data collection, interpretation of data for the work and critical revision for important intellectual content. PM: study design, data collection, data analysis, interpretation of data and critical revision for important intellectual content. JP: study design, data analysis, interpretation of data for the work and critical revision for important intellectual content. CJ, HLK: study design, data analysis and critical revision for important intellectual content. HLE: study design, concept development, data collection and analysis, interpretation of data for the work and critical revision for important intellectual content. All authors approved the final version to be published. All authors had full access to all the data in the study and had final responsibility for the decision to submit for publication.

Funding This work was supported by the Global Health and Vaccination Research (GLOBVAC) programme at the Research Council of Norway (project number 228203), the Laerdal Foundation and the Saving Lives at Birth Grand Challenges.

Disclaimer The external funding sources had no role in study design, data collection, data analysis, data interpretation, writing of the report, or in the decision to submit the paper for publication.

Competing interests None declared.

Patient consent for publication Not required.

Ethics approval The National Institute for Medical Research (NIMR) and the Ministry of Health in Tanzania (the HBB CQI programme Ref NIMR/HQ/R.8a/Vol IX/1247 and the Safer Births project Ref NIMR/HQ/R8a/Vol IX/1434), and by the Regional Committee for Medical and Health Research Ethics, Western Norway (Ref 2009/302 and Ref number 2013/110/REK).

Provenance and peer review Not commissioned; externally peer reviewed.

Data availability statement Data are available upon reasonable request.

Open access This is an open access article distributed in accordance with the Creative Commons Attribution Non Commercial (CC BY-NC 4.0) license, which permits others to distribute, remix, adapt, build upon this work non-commercially, and license their derivative works on different terms, provided the original work is properly cited, appropriate credit is given, any changes made indicated, and the use is non-commercial. See: http://creativecommons.org/licenses/by-nc/4.0/.

\section{REFERENCES}

1. Wang $\mathrm{H}$, Liddell CA, Coates MM, et al. Global, regional, and national levels of neonatal, infant, and under-5 mortality during 1990-2013: a systematic analysis for the global burden of disease study 2013 . The Lancet 2014;384:957-79.

2. WHO. Who fact sheet 178 children: reducing mortality 2016 .

3. Blencowe $\mathrm{H}$, Cousens $\mathrm{S}$, Jassir FB, et al. National, regional, and worldwide estimates of stillbirth rates in 2015, with trends from 2000 : a systematic analysis. Lancet Glob Health 2016;4:e98-108.

4. Vel and trend in child mortality report 2013, estimates developed by the Inter-agency group for child mortality estimation. PG 1113. Available: http://www.who.int/maternal_child_adolescent/ documents/levels_trends_child_mortality_2013.pdf?ua=1 [Accessed January 2019].

5. UNICEF, who, world bank group and United nations. Levels and trends in child mortality, report 2017, October 18, 2017. Available: https://www.unicef.org/publications/index_101071.html [Accessed Jan 2019].

6. Newborns: reducing mortality, who fact sheet 333 Jan, 2016. Available: http://www.who.int/mediacentre/factsheets/fs333/en/ [Accessed January 2019].

7. United Nations Children's Fund (UNICEF) report, 2018 EVERY CHILD ALIVE The urgent need to end newborn deaths. Available: https:// www.unicef.org/publications/index_102640.html [Accessed January 2019].

8. Darmstadt GL, Shiffman J, Lawn JE. Advancing the newborn and stillbirth global agenda: priorities for the next decade. Arch Dis Child 2015;100(Suppl 1):S13-S18.

9. Singh K, Brodish P, Suchindran C. A regional multilevel analysis: can skilled birth attendants uniformly decrease neonatal mortality? Matern Child Health J 2014;18:242-9.

10. Ersdal HL, Mduma E, Svensen E, et al. Early initiation of basic resuscitation interventions including face mask ventilation may reduce birth asphyxia related mortality in low-income countries: a prospective descriptive observational study. Resuscitation 2012;83:869-73.

11. Msemo G, Massawe A, Mmbando D, et al. Newborn mortality and fresh stillbirth rates in Tanzania after helping babies breathe training. Pediatrics 2013;131:e353-60.

12. Mduma $E$, Ersdal $H$, Svensen $E$, et al. Frequent brief on-site simulation training and reduction in 24-h neonatal mortality--an educational intervention study. Resuscitation 2015;93:1-7.

13. Langli Ersdal H, Mduma E, Svensen E, et al. Intermittent detection of fetal heart rate abnormalities identify infants at greatest risk for fresh stillbirths, birth asphyxia, neonatal resuscitation, and early neonatal deaths in a limited-resource setting: a prospective descriptive observational study at Haydom Lutheran Hospital. Neonatology 2012;102:235-42.

14. Ersdal HL, Mduma E, Svensen E, et al. Birth asphyxia: a major cause of early neonatal mortality in a Tanzanian rural hospital. Pediatrics 2012;129:e1238-43.

15. Mduma ER, Ersdal H, Kvaloy JT, et al. Using statistical process control methods to trace small changes in perinatal mortality after a training program in a low-resource setting. Int $J$ Qual Health Care 2018;30:271-5

16. Goudar SS, Somannavar MS, Clark R, et al. Stillbirth and newborn mortality in India after helping babies breathe training. Pediatrics 2013;131:e344-52.

17. Bellad RM, Bang A, Carlo WA, et al. A pre-post study of a multicountry scale up of resuscitation training of facility birth attendants: does helping babies breathe training save lives? BMC Pregnancy Childbirth 2016;16:222.

18. Watkins RE, Eagleson S, Veenendaal B, et al. Applying cusumbased methods for the detection of outbreaks of Ross River virus disease in Western Australia. BMC Med Inform Decis Mak 2008;8:37

19. Bottle A, Aylin P. Intelligent information: a national system for monitoring clinical performance. Health Serv Res 2008;43:10-31.

20. Mduma ER, Gratz J, Patil C, et al. The etiology, risk factors, and interactions of enteric infections and malnutrition and the consequences for child health and development study (MAL-ED): description of the Tanzanian site. Clin Infect Dis 2014;59(suppl_4):S325-30.

21. Ersdal HL, Vossius C, Bayo E, et al. A one-day "Helping Babies Breathe" course improves simulated performance but not clinical management of neonates. Resuscitation 2013;84:1422-7.

22. Mdoe PF, Ersdal HL, Mduma ER, et al. Intermittent fetal heart rate monitoring using a fetoscope or hand held Doppler in rural Tanzania: a randomized controlled trial. BMC Pregnancy Childbirth 2018;18:134.

23. Mdoe PF, Ersdal HL, Mduma E, et al. Randomized controlled trial of continuous Doppler versus intermittent fetoscope fetal heart rate monitoring in a low-resource setting. Int $J$ Gynecol Obstet 2018:143:344-50. 
24. Kamala BA, Ersdal HL, Dalen I, et al. Implementation of a novel continuous fetal Doppler (Moyo) improves quality of intrapartum fetal heart rate monitoring in a resource-limited tertiary hospital in Tanzania: an observational study. PLoS One 2018;13:e0205698.

25. Thallinger M, Ersdal HL, Francis F, et al. Born not breathing: a randomised trial comparing two self-inflating bag-masks during newborn resuscitation in Tanzania. Resuscitation 2017;116:66-72.

26. Massawe A, Kidanto HL, Moshiro R, et al. A care bundle including antenatal corticosteroids reduces preterm infant mortality in Tanzania a low resource country. PLoS One 2018;13:e0193146.

27. Lovegrove J, Valencia O, Treasure T, et al. Monitoring the results of cardiac surgery by variable life-adjusted display. The Lancet 1997;350:1128-30.

28. Zeng L, Pham H. Quality and reliability management and its applications. Springer series in reliability engineering. 1st edn, 2016.

29. Lie RT, Heuch I, Irgens LM. A new sequential procedure for surveillance of Down's syndrome. Stat Med 1993;12:13-25.

30. Steiner SH, Cook RJ, Farewell VT, et al. Monitoring surgical performance using risk-adjusted cumulative sum charts. Biostatistics 2000;1:441-52.
31. Gandy A, Kvaløy Jan,Terje, Kvaløy JT. spcadjust: an R package for adjusting for estimation error in control charts. $R J$ 2017;9:458-76.

32. Sialubanje C, Massar K, Hamer DH, et al. Reasons for home delivery and use of traditional birth attendants in rural Zambia: a qualitative study. BMC Pregnancy Childbirth 2015;15:216.

33. Ersdal HL, Eilevstjønn J, Linde JE, et al. Fresh stillborn and severely asphyxiated neonates share a common hypoxic-ischemic pathway. Int J Gynecol Obstet 2018;141:171-80.

34. Makene CL, Plotkin M, Currie S, et al. Improvements in newborn care and newborn resuscitation following a quality improvement program at scale: results from a before and after study in Tanzania. BMC Pregnancy Childbirth 2014;14:381.

35. Eblovi D, Kelly P, Afua G, et al. Retention and use of newborn resuscitation skills following a series of helping babies breathe trainings for midwives in rural Ghana. Glob Health Action 2017;10:1387985.

36. Mukhtar-Yola M, Audu LI, Olaniyan O, et al. Decreasing birth asphyxia: utility of statistical process control in a low-resource setting. BMJ Open Qual 2018;7:e000231. 